\title{
Rekonstruktion der biographischen Genese einer Bewährungsfigur - Ein Beitrag zu einem sozialisationstheoretischen Begriff der Bewährung
}

Boris Zizek (Universität Mainz, z.Z. Harvard University)

\section{Fokus und Fragestellung}

Ziel des vorliegenden Beitrags ist es, exemplarisch die biographische Genese einer Bewährungsfigur zu rekonstruieren und auf diesem Wege zur weiteren Differenzierung dieses sozialisationstheoretischen Begriffs beizutragen, wobei dieses Bestreben seinerseits im Dienste der weiteren Ausbuchstabierung einer anvisierten sozial- und erziehungswissenschaftlichen Theorie der Bewährung steht.

$\mathrm{Zu}$ dieser Unternehmung habe ich bisher eine kulturhistorische Untersuchung zu spezifisch modernen Bewährungsmustern (Zizek 2012) durchgeführt. Es konnte gezeigt werden, dass die Subjekte Bewährungsfiguren ausbilden, die eine den kulturspezifischen Formen des Zusammenlebens entsprechende, funktionale Bewährung ermöglichen. Die Folgerung war, dass diesen spezifischen Ausformungen ein universaler Bewährungsdrang zugrunde liegen muss und es aufschlussreich sein könnte, den Menschen als einen Bewährungssucher in den Blick zu nehmen.

In einem um Systematisierung bemühten Aufsatz ging es mir darum, dieses Konzept als solches zu akzentuieren und durch eine Kontrastierung mit klassischen theoretischen Konzepten des Subjekts und der Motivation weiter zu schärfen (2015a). Es war mein Bestreben, die zentrale Stellung des Begriffs der Bewährung aufzuzeigen, die diesem sowohl für die Grundlagentheorie der Sozialwissenschaft, als auch für die Diagnose aktueller sozialisations- und professionalisierungstheoretischer Problemstellungen zukommt. ${ }^{1}$

${ }^{1}$ Ich möchte auch kurz darauf hinweisen, dass Olaf Behrendt und ich zu der Fragestellung Autonomie und Bewährung zwei interdisziplinäre Tagungen an den Universitäten Mainz und Frankfurt am Main (auf Einladung von Claudia Peter) durchgeführt haben und eine Veröffentlichung mit Beiträgen aus der Sozial- und Erziehungswissenschaft, der Schul- und Sonderpädagogik in der Vorbereitung ist. 
Einem nochmals anders gelagerten Test habe ich das Konzept im Kontext des Themenfeldes Adoleszenz, Schule, Unterricht und pädagogische Professionalität ausgesetzt $(2013,2015 b)$. Einerseits ging es mir darum, ob und wie man störende Schüler aus der bewährungstheoretischen Perspektive betrachten muss und welche Schlussfolgerungen aus dieser für die pädagogische Professionalität zu ziehen sind. Meine Ansichten dazu habe ich als Maximen einer positiven Pädagogik akzentuiert. Die Rekonstruktion von typischen Bewährungsmustern von Schülern wie dem Klassenclown, der KlassensprecherIn und dem Mauerblümchen, habe ich im Kontext meiner Mitarbeit im von Robert Selman und Helen Haste geleiteten China Lab der Harvard Graduate School of Education um eine vergleichende Rekonstruktion von Bewährungsfiguren von chinesischen 11 Klässlern aus städtischen (Shanghai) und ländlichen Schulen erweitert.

Im Folgenden soll es nun darum gehen, die Herausbildung einer Bewährungsfigur exemplarisch zu rekonstruieren. Es handelt sich um einen ersten Versuch, der gewiss einiges unberücksichtigt lassen wird, was wichtig gewesen wäre. Sollte es mir gelingen, die Idee, die ich mit dem Begriff in den Blick nehme, kenntlich zu machen und weiter zu konturieren, dann wäre das angestrebte Ziel erreicht.

Zieht man explorativ zu Kontrastierungszwecken ${ }^{2}$ weitere Begriffe heran, die gebraucht werden, um Motivationen $\mathrm{zu}$ benennen und $\mathrm{zu}$ charakterisieren, dann haben Bewährungsfiguren mit Bedürfnissen, die befriedigt werden, den basalen Charakter des motivierenden Inhalts gemeinsam. Während der Begriff des Bedürfnisses jedoch etwas benennt, was man tendenziell als gegeben annehmen muss und was im Sinne von Grundbedürfnissen entsprechend der Tendenz nach auch für alle gleichermaßen zutrifft, verweist der Begriff der Bewährungsfigur auf einen motivierenden Inhalt, der gewachsen, sich erfahrungsabhängig herausgebildet hat. Entsprechend stehen Interessen Bedürfnissen näher, wobei sie differenzierter sind und gezielt verfolgt werden. Mit dem Begriff der Ideen hat Max Weber in seinen wirtschaftsethischen Untersuchungen bekanntlich den Interessen einen Motivationstypus entgegengesetzt, der auf Beweggründe zielt, die sich nicht aus der gegenwärtigen Situation oder Lage heraus erklären lassen, sondern geschichtlich, erfahrungsabhängig gewachsene Sinnstrukturen sind. ${ }^{3}$ Diese Charakteristik teilen Bewährungsfiguren mit ihnen, wobei sie im Vergleich zu Ideen, die bei Weber kollektivspezifische

2 Die folgende Auswahl ist weder vollständig noch systematisch, sondern ist an der Hervorhebung von spezifischen Aspekten von Bewährungsfiguren orientiert.

${ }^{3}$ Anthony Giddens weist in seiner Einleitung zur englischsprachigen Ausgabe der Protestantischen Ethik auf die zentrale Stellung der Geschichte im Weberschen Zugang zu sozialen Phänomenen wie dem ökonomischen Handeln hin (2001). 
Motive benennen, fallspezifische Beweggründe fokussieren, also durchaus an ihnen vorgängige Ideen anknüpfen. Wesentlich ist jedoch, dass sie darüber hinaus Aspekte und Akzentuierungen enthalten müssen, die der Träger als nur sie/ihn auszeichnend auffasst. Kontrastiert man Bewährungsfiguren mit Selbstbildern dann wird deutlich, dass letztere der Deutungsebene zugehören, Bewährungsfiguren hingegen vorreflexiv, dem Subjekt in der Regel nicht explizit bewusst sind, sondern mehr den Kern des Selbstgefühls im Sinne Eriksons bilden. Man könnte sie entsprechend auch als identitätsrelevante Habitusfigurationen paraphrasieren, womit auch ihr hoher Internalisierungsgrad angesprochen wäre. Ich gehe nämlich davon aus, dass Bewährungsfiguren zwar einerseits in neuen sozialen Kontexten und im Zuge der Persönlichkeitsentwicklung modifiziert, mit neuen Aspekten versehen, erweitert und im Sinne des Hegelschen Begriffs des Aufhebens in Haltungen mit einem höheren Niveau der Komplexität integriert werden, andererseits bilden sie aber der Idee nach dauerhaft den Kern oder eine wesentliche Tendenz möglicher Identitätsformationen bilden.

\section{Kurze Vergegenwärtigung des Konzepts des Menschen als Bewährungssucher}

Wie muss man sich diesen universalen Bewährungsdrang vorstellen, von dem oben bereits die Rede war? In ,Warum wir kooperieren' weist Michael Tomasello auf eine intrinsisch motivierte uneingeschränkte Kooperationsbereitschaft von deutlich unter 2jährigen hin. Kooperation setzt sich zusammen aus der Fähigkeit, „Ziele anderer ... zu erkennen“ (Tomasello 2010, 22), und dem „altruistischen Motiv“ (ebd.), ihnen dabei auch helfen zu wollen. Diese Kooperationsbereitschaft ist zunächst uneingeschränkt, die Kinder unterscheiden noch nicht, wem sie helfen und wem nicht (vgl. ebd., 19). Sie ist außerdem intrinsisch motiviert (vgl. ebd., 23), „Belohnungen oder Ermutigungen durch die Eltern [steigern] das hilfsbereite Verhalten der Kinder nicht" (ebd., 22).

Diese intrinsisch motivierte, uneingeschränkte Kooperationsbereitschaft ist ein zentrales Moment des universalen Bewährungsdrangs. Bei Erikson findet sich eine wesentliche Ergänzung. In seinem Aufsatz Ich-Entwicklung und geschichtlicher Wandel thematisiert Erikson implizit einen universalen Bewährungsdrang. Er weist darauf hin, der „Drang“ des Kindes, „... den Akt des Laufens zu wiederholen und zu vervollkommnen ..." sei nicht nur auf die „... libidinöse[r] Lust im Sinne Freuds Bewegungs-Erotik ...“ oder auf das „... Bedürfnis nach bestmöglicher Beherrschung im Sinne von I. Hendricks Arbeitsprinzip ...“ (Erikson 1973, 17) zurückzuführen. Das Kind „... entdeckt auch, daß es mit der neuen Körperhaltung einen neuen Status bekommen kann: ,einer der Laufen kann' mit all den Nebenbedeutungen, die diese Kunst innerhalb des Lebensplans seiner Kultur besitzen mag: einer der vorwärts 
kommen wird' oder ,einer der aufrecht dastehen wird' oder auch ,einer der zu weit gehen wird“" (ebd.). Der „Drang“ des Kindes zur Vervollkommnung des Akts des Laufens speist sich auch aus der Entdeckung, dass damit eine wertvollere Position in der Gemeinschaft näher rückt. Das Kind merkt, dass es sich mit dieser Fähigkeit etwa als „einer der aufrecht dastehen wird“ einst in einem höheren Maße bzw. echt bewähren können wird.

Eine weitere Evidenz dafür, dass es bereits Kleinkindern darauf ankommt, sich durch die Bewältigung echter Aufgaben in einer Gemeinschaft nützlich zu machen, sich also zu bewähren, bietet folgende Schilderung der Leiterin eines Kindergartens, die sie meiner Frau und mir bei der Anmeldung unseres Sohnes beiläufig erzählte. Ihr kleines Eckbüro befand sich am Ende des Kindergartens. Die Kinder besuchten sie dort sehr gerne. Sie würden ihr auch immer unermüdlich helfen, wenn sie ihnen etwa Papiere zum Lochen für die Ordner gäbe. Allerdings verlören sie sehr schnell die Lust, wenn sie ihnen mangels solcher Arbeiten unechte, künstliche Aufgaben zu erledigen gäbe. Diese Erzählung macht anschaulich, dass Kinder schon sehr früh zwischen echten und unechten Aufgaben unterscheiden und dass nur echte Aufgaben sie in einem besonderen Maße motivieren. Bewährung bedeutet also einen echten Beitrag zum Gemeinwohl. Diesen „Drang“, der in dem von Erikson hervorgehobenen Streben nach Aneignung künftiger Bewährungspositionen greifbar wird und der sich motivational ausschließlich aus echten Aufgaben zu speisen scheint, würde ich, da er bereits bei Kleinkindern auftritt, als universalen Bewährungsdrang bezeichnen (vgl. Zizek 2012). Dieser erfüllt und manifestiert sich auf eine identitätsrelevante Weise in Bewährungsfiguren. Anhand dieser sozialen Kontur erkennt, fühlt man sich als einzigartiges und wertvolles Mitglied einer Gemeinschaft.

Der universale Bewährungsdrang erscheint als die Freilegung einer vorgängigen sozialen Motivierung. Damit richtet sich das Konzept gegen die klassischen Subjektkonzepte der Psychoanalyse, der Wirtschafts- und der Sozialwissenschaften, also den homo oeconomicus, den homo sociologicus und den Menschen Freuds. In ihnen ist soziale Motivierung stets als ein sekundäres oder Mit-Motiv konzipiert. Damit entspricht das Konzept des Menschen als Bewährungssucher der sozialisationstheoretischen Logik der Rekonstruktion vorgängiger Einbettungen und Prozesse und der Herausarbeitung der sozialen Natur des Menschen und ist von daher neben dem Konzept der Anerkennung als ein spezifisch sozialisationstheoretisches Motivationskonzept zu begreifen. 


\section{Der, der sich im Gegensatz zu seinen Familienmitgliedern neue Kontexte erfolgreich aneignet - Rekonstruktion der Genese einer Bewährungsfigur}

Peter, dessen Eltern vor seiner Geburt aus dem Ausland eingewandert sind, war zum Zeitpunkt des Interviews 20 Jahre alt. Er hat einen jüngeren Bruder, der das Gymnasium nicht geschafft hat, während er selbst gerade sein Studium mit einem Magisterstudiengang im Bereich der Sozial- und Geisteswissenschaften begonnen hatte. Aus Gründen der Anonymisierung möchte ich hier keine weiteren Kontextinformationen anführen, zumal sie zum Fokus der Untersuchung nichts Wesentliches beitragen würden.

Nach einem kurzen erläuternden Vorgespräch forderte ich Peter dazu auf, mir seine Lebensgeschichte zu erzählen. Als er nachfragte, um eine Präzisierung zum Anfang der Narration bat, legte ich ihm nahe, frei assoziierend zu erzählen, was ihm zu seiner Lebensgeschichte gerade als erstes einfiele, wodurch die Erzählung von der Erwartung strenger Chronologie entlastet wurde. Peter rahmte seine lebensgeschichtliche Narration dann mit einer konjunktivischen Konstruktion gleichsam als Gedankenexperiment. Er nahm also die Herausforderung, die mit dem Modus freier Assoziation verbunden ist, selbstbewusst an.

„Ähm..gut... ich würd ähm...anfang` mit“.

Durch ihre entlastende Unverbindlichkeit eröffnet diese Rahmung einerseits einen experimentier- und entwurfsfreundlichen Raum subjektiver Entfaltung. Würde man sie jedoch nicht produktiv nutzen, dann fiele sie andererseits zu einer modisch unverbindlichen und wohl auch etwas selbstverliebten Attitüde des Bewusstseins der Vorläufigkeit aller Ansichten zusammen.

Mit „es gab so'n kleinen Umbruch in mein'm Leben ..." leitet Peter dann lapidar die Erzählung von der Pleite des Geschäfts seiner Eltern und den daraus resultierenden Folgen für die Familie ein. Mit der gewählten Weise der Schilderung durch „es gab“ werden die Ereignisse als unbeeinflussbar eingeführt und auch nicht in den Fokus einer Erklärungssuche gerückt. Das Erzählte erhält dadurch den Charakter von etwas, das gleichsam hinzunehmen war. Dies dürfte der damaligen Erlebensperspektive durchaus entsprochen haben, so dass hier einerseits ein kindlicher Blick auf Ereignisse eingerichtet wird. Indem Peter von einem „Umbruch in mein'm Leben..." spricht, es also als ein von seiner Familie getrenntes thematisiert, nimmt er andererseits eine Bedeutungsaufladung seiner kindlichen Perspektive vor, die hier als selbstbewusst und aktiv akzentuiert wird. Die Ereignisse haben ihn in seiner schon damals ausdifferenzierten Lebensführung belastet.

Es handelt sich hier natürlich um eine Retrospektive, doch könnte diese aktivische Akzentuierung durchaus an ein Gefühl anknüpfen, dass sich 
durch den „Umbruch" tatsächlich schon damals eingestellt haben könnte, und dem eine gleichsam verfrühte Exzentrisierung seiner Wahrnehmung zugrunde lag. Dieser Umbruch hat Peter damals vielleicht so stark getroffen, dass es ihn ein erstes Mal aus der selbstverständlichen kindlichen alltäglichen Eingebettetheit gerückt hat. Und diese Position scheint sich aus noch zu klärenden Gründen darüber hinaus auch zu einem gewissen Grad verfestigt zu haben, so dass er in seiner Retrospektive daran anknüpft.

In der Attribuierung des Umbruchs als „so`n kleinen“ vollzieht Peter jedoch im selben Atemzug eine sarkastische Distanznahme. Nachdem auf der einen Seite eine einschneidende Erfahrung greifbar wurde, wird das Ganze nun heruntergespielt, es ist nicht der Rede wert. Auf diese Weise entsteht ein rasantes Wechselspiel von Perspektiven, einem Sichöffnen folgt ein Sichzurückziehen auf dem Fuße. Der Rückzug, der durch Entdramatisierung der gerade erst eröffneten Thematisierung von wunden Punkten sogleich wieder wegwischt wird, scheint wie ein Selbstschutz zu fungieren und das Sichöffnen überhaupt zu ermöglichen. Ein Gegenüber würde es jedoch schwer haben, mit diesem schnellen Wechselspiel aus Angebot einer Öffnung und dem plötzlichen Abzug des Interesses, zurecht zu kommen.

Mit dem bestimmten Artikel "das", der sich in der nun folgenden Erläuterung „...das hatte mit äh dem Geschäft meiner Eltern zu tun..." summarisch auf das eben Angesprochene bezieht, wird die nun einsetzende Thematisierung von Gründen für den Umbruch gleich zu Beginn wieder eingenebelt. Es deutet sich hier an, dass die Eltern diesen einschneidenden Umbruch verursacht haben, wobei sich Peter hier sträubt, seine Meinung klar auszusprechen. Vielmehr vernebelt er die Akteursposition seiner Eltern, indem er das "Geschäft meiner Eltern" an die Stelle der Ursache setzt. Wie verärgert über das uneinsichtige Verhalten eines Kind sieht Peter hier davon ab, die Eltern als Verursacher beim Namen zu nennen. Das impliziert eine starke Distanzierung und eine im Grunde immer noch anhaltende Verärgerung, wobei er die Eltern meint schonen zu müssen und ihnen mit dieser überheblichen Attitüde die Zurechenbarkeit abspricht.

Das Geschäft der Eltern, „...das ist dann irgendwann kaputt gegangen..... Peter rückt die Eltern nun erneut infantilisierend in die Position von Kindern, die solange Blödsinn machen oder untätig bleiben, bis etwa „kaputt“ geht. Mit „irgendwann“ wird darauf hingewiesen, dass das Geschäft wohl schon länger schlecht lief, bevor es dann geschlossen werden musste. Es wird die Einschätzung nahe gelegt, dass die Eltern taten- und ideenlos dem Niedergang zugeschaut haben.

Diese Logik der infantilisierenden Generationenumkehr, der zufolge Peter die Unfähigkeit seiner Eltern ertragen musste, setzt sich fort. Erneut vernebelt er nachsichtig die ursächliche Rolle seiner Eltern, wenn er eine aus der Pleite des Geschäfts resultierende Veränderung nur temporal verortet, „....und dann gabs so' n ganz krassen biographischen Umbruch auch ,...". Mit dem terminus 
technicus biographischer Umbruch akzentuiert er seine distanzierte Perspektive. Man könnte annehmen, dass seine immer noch anhaltende Enttäuschung und Wut über das Versagen seiner Eltern ihn seinerzeit auf Distanz gebracht, exzentrisiert haben, so dass er frühreif die Idee entwickelt hatte, sein Leben selbst in die Hand zu nehmen.

Ein solcher Eindruck kann nun sicherlich im Sinne einer Selbstcharismatisierung erhebend und beflügeln wirken, er birgt kehrseitig jedoch auch die Gefahr, dass man die einschränkenden Umstände des Lebens, mit denen es die Eltern sicherlich auch zu tun hatten, aus den Augen zu verlieren geneigt sein kann und sich selbst über- und andere unterschätzt. Eine Einschränkung der Wahrnehmung deutet sich hier bereits dadurch an, dass Peter die Geschäftspleite, die für die Eltern dramatisch gewesen sein muss, gegenüber dem Folgenden als einen kleinen Umbruch charakterisiert. Peter spezifiziert diesen eigentlichen, „krassen“ Umbruch dann als Umzug aus einem großen Haus in eine kleine Wohnung. „...das heißt wir sind umgezogen aus dem großen Haus in ne kleine Wohnung...". Das Erleben wird durch die Opposition von groß und klein akzentuiert. Das Haus bot sicherlich mehr Platz fürs Spielen und subjektive Entfaltung, die Wohnung hingegen bedeutete eine diesbezügliche Verengung. Während man durch den bestimmten Artikel "dem" vor „Haus" dieses gleichsam vor Augen hat, wird die Wohnung, obwohl sie lebensgeschichtlich näher liegt, durch den unbestimmten und abgekürzten Artikel vergleichsweise unlebendig und unpersönlich vergegenwärtigt.

Anschließend betont Peter nun mit Nachdruck die Gleichzeitigkeit dieses beruflichen und privaten Abstiegs der Familie, der in der Verengung des Zuhauses seinen physischen Ausdruck erfährt, auf der einen Seite und seiner Einschulung auf der anderen Seite.

„...äh das war genau zur Zeit meiner Einschulung in die Grundschule..."

Die ausführliche Korrektheit der Erwähnung der Einschulung markiert diese als bedeutsames Ereignis und wertet damit auch ihn als jemanden auf, auf den man Rücksicht nehmen sollte. Diese retrospektive, ausführliche Wertschätzung der „Einschulung in die Grundschule“ lässt eine gelungene Meisterung erwarten. Man kann an dieser Stelle festhalten, dass Peter die Krisen seiner Eltern zu kleinen Umbrüchen abwertet und seine eigenen zu großen Herausforderungen aufwertet.

Der Schulbeginn als Heraustreten aus dem Elternhaus in einen sozialen Raum mit einer neuen dezentrierenden sozialen Logik wie sie im egalisierenden Leistungsprinzip und dem Prinzip spezifischer Sozialbeziehung zum Ausdruck kommt, bedeutet für das Kind eine Herausforderung. Deren Bewältigung wurde durch den Wegfall des großen Hauses als vertrautem Rückzugsort erschwert. Darauf will Peter hier hinweisen. Es war gleichsam eine doppelte Herausforderung, wobei er durch die Dramatisierung der Gleichzeitigkeit 
hervorhebt, es trotz dieser geschafft zu haben. Es wird sich im Folgenden zeigen, dass Peter in dieser Verdichtung bereits implizit auf die zentrale Logik seiner sich in der Folge weiter sättigenden und ausgestaltenden Bewährungsfigur aufmerksam gemacht hat: Ich habe mir im Gegensatz zu den gegenteiligen Tendenzen in meiner Familie trotz erschwerter Bedingungen allein eine neue Umgebung erfolgreich angeeignet.

Peter berichtet in der Folge, dass sein Vater seit der Geschäftspleite nur noch Tippgemeinschaften organisiert und auch Geld unterschlagen habe. Fasst man das Glücksspiel in einer ersten Annäherung im Kontrast zu realitätsgerechter Auseinandersetzung als eine manipulative Wirklichkeitsbewältigung auf, da ihm die Hoffnung auf eine rasante Wende der aktuellen Lebenssituation zugrunde liegt, dann scheint der von Peter oben angedeutete unbewegliche Umgang der Eltern mit dem drohenden Niedergang des Geschäfts zumindest beim Vater einen habituellen Charakter zu haben. Statt sich Neuem zu öffnen, hängt man im Hoffen auf eine alles verändernde Wende im Grunde Verlorenem nach. Dies schätzt auch Peter so ein. Sein Vater lerne kein Deutsch, was seine realen Möglichkeiten verbessern würde, vielmehr sei er der Meinung, „irgendwann wird schon alles wieder gut“. Seine Mutter, die als einzige arbeitet, solle sich daher "davon loslösen... und endlich mal ihr eigenes Leben“" zu leben versuchen.

Peter hat sich fortan "wesentlich weniger zu Hause" aufgehalten. Aus der biographischen Erfahrung, in der das Scheitern der Eltern mit der räumlichen Verengung zusammengebunden wird, scheint sich eine charakteristische habituelle Bewegungsfigur herausgebildet zu haben, die man als Flucht-aus-sich-verengender-Erstarrung paraphrasieren könnte und die mit der oben explizierten Bewährungsfigur harmoniert.

Ich war viel draußen. Ich bin immer noch viel draußen ich bin sobald die Sonne aufgeht bin ich eigentlich draußen und spiele und mach Sport oder auch nicht und lieg einfach draußen ... weil ich Platz brauche und deswegen dann nach draußen geh weil ich drinnen nich genug Platz habe ...Und lern auch da lieber. Mach alles andre dort lieber.

Einerseits hat die erstarrende Unbeweglichkeit der Eltern tatsächlich eine räumliche Verengung zur Folge, andererseits scheint die Bewegungsfigur im Sinne einer Metapher das Erfahrungsurteil ästhetisch $\mathrm{zu}$ gestalten, dass Transformationsvermeidung mit Verengung des Handlungsspielraumes bezahlt werden muss. Der Fall beschreibt die Lösung aus der sich immer weiter verengenden Familienwelt als aktive Abkapselung.

Ich hab selber das Gefühl über mich selbst, dass ich mich davon einigermaßen abkapseln konnte während ich so dass die andern drei so total ineinander involviert und verstrickt sind und da nie wieder rauskomm: 
Peters dynamische Bewegungsfigur, die auch einen entscheidenden Anteil an der Differenz zur Entwicklung des Bruders zu haben scheint, der das Gymnasium nicht schafft und rebellierend verstrickt bleibt, rückt ihn in die oben bereits thematisierte exzentrische Perspektive, von der aus er auf seine Familie blickt.

Peter berichtet im Verlauf des Interviews, dass ihn die Auseinandersetzung mit den Familienproblemen zu seiner Studienwahl geführt habe. Er möchte Sinnzusammenhänge verstehen und in seiner späteren beruflichen Tätigkeit anderen Menschen therapeutisch helfen. Seine Studienmotivation kann nun scheinbar nahtlos an seine Bewährungsfigur anknüpfen, der folgend er sich aus verengender Erstarrung lösend erfolgreich neue Umgebungen anzueignen vermag. Er berichtet:

Ich wollt schon immer mit Professoren reden, diskutier`n weil ich ähm ich wollt immer 'ne Stufe höher auf, Gymnasium hat’s mir irgendwann nich mehr gereicht weil dann war`ns immer die Standardantworten.

Die Professoren und die Institution Universität verheißen ihm neue Perspektiven und Nichtfestgefahrenheit. Sein Selbstvertrauen scheint dabei gleichsam unerschütterbar. Ist hier das selbstcharismatisierende Potential der Bewährungsfigur angesprochen, so wird an einer späteren Stelle auch das inhärente Risikopotential nochmals deutlich greifbar. In folgendem Abschnitt antwortet Peter auf die Frage, was sich an seinem Leben seit der Schulzeit verändert habe:

Also seit dem Tag an dem ich mein Abitur überreicht habe hatt' ich die wahnsinnige Sehnsucht nach der Universität Das heißt, ich hätt’ am liebsten gleich im Anschluss am nächsten Tag wär ich an die Universität gegangen...

Neben dem in der "Sehnsucht nach der Universität" erneut zum Ausdruck kommenden Durst nach Perspektiverweiterung bringt die Fehlleistung, sich das Abitur selbst überreicht zu haben, hier nochmals deutlich zum Ausdruck, dass Peter Autoritäten nur mit einer gewissen Ungeduld anerkennt, um sie auf der nächsten Stufe hinter sich lassen zu wollen. Gleichsam autoritätenlöschend überreicht er sich das Abitur selbst. Die Nichtanerkennung der erbrachten Leistung der ihn sozialisierenden Akteure zieht sich durch das gesamte Interview.

\section{Aspekte einer Bewährungsfigur}

Im vorliegenden Fall leistet die rekonstruierte Bewährungsfigur auf sehr anschauliche Weise eine Konturierung des Selbst innerhalb der Familie. Diese erste lebensgeschichtliche Selbstkonturierung findet über eine den Fall positiv 
auszeichnende, besondere Eigenschaft statt ("Ich meistere schwierige Herausforderungen besser als..."). Die Bewährungsfigur hat dabei den Charakter eines ersten intuitiven lebensgeschichtlichen Resümees aus einer egozentrierten Perspektive heraus (Was ist mein Beitrag und Stellung in dieser Welt?).

Es wurde aufgezeigt, dass diese Bewährungsfigur nach ihrer ersten Kristallisation in der Folge eine sukzessive Sättigung und Ausgestaltung erfahren hat, indem sich allmählich weitere strukturhomologe Routinen um sie gruppierten. Weiter wurde hervorgehoben, dass die Bewährungsfigur die zentrale Quelle der Selbstcharismatisierung, des Vertrauens in die eigenen Fähigkeiten und Problemlösungsvorschläge, bildet. Neben der Selbstvergewisserung, deren Notwendigkeit der humanspezifischen Lebenssituation geschuldet ist, ist die zweite wesentliche Funktion von Bewährungsfiguren also die Selbstcharismatisierung ("Ja, das kann ich, das bin ich"). Letztere ist bei dem vorliegenden Fall besonders stark ausgeprägt, insofern sich Peter in einer impliziten Generationenumkehr seinen Eltern nicht nur ebenbürtig, sondern in Bezug auf die Lebensbewältigung sogar überlegen fühlt. In diesem Zusammenhang wurde deutlich, dass die Besonderung und Bestärkung, die man aus Bewährungsfiguren ziehen kann, dazu verleiten können, die Leistung anderer abzuwerten, um den eigenen Beitrag vorteilhafter zu konturieren (Ich kann was sie gar nicht hinkriegen) oder die eigenen Aktionen zu rationalisieren.

Die Interviewanalyse hat schließlich darauf aufmerksam gemacht, dass die Bewährungsfigur in Peters Bildungskarriere eine produktive, komplexitätserhöhende Transferierung auf neue Felder der Bewährung erfährt. Dies muss, wie bereits angedeutet, nicht immer gelingen, so dass es etwa im therapeutischen und sozialpädagogischen Bereich fruchtbar sein könnte, Störungen oder deviantes Verhalten als Formen selbstschädigender Bewährung in den Blick zu nehmen.

\section{Literatur}

Erikson, E. H. 1973. Identität und Lebenszyklus. Frankfurt am Main: Suhrkamp. Giddens, A. 2001. Introduction. In Weber, M. (Hrsg.), The Protestant Ethic and the Spirit of Capitalism. London - New York: Routledge.

von Hentig, H. 2006. Bewährung. Von der nützlichen Erfahrung, nützlich zu sein. München: Hanser.

Oevermann, U. 1995. "Ein Modell der Struktur von Religiosität. Zugleich ein Strukturmodell von Lebenspraxis und von sozialer Zeit". In WohlrabSahr, M. (Hrsg.), Biographie und Religion. Zwischen Ritual und Selbstsuche. Frankfurt am Main: Campus: 27-102. 
-_- 1996. "Theoretische Skizze einer revidierten Theorie professionalisierten Handelns". In Combe, A. \& Helper, W. (Hrsg.), Pädagogische Professionalität. Untersuchungen zum Typus pädagogischen Handelns. Frankfurt am Main: Suhrkamp: 70-182.

- — . 2003. "Strukturelle Religiosität und ihre Ausprägungen unter Bedingungen der vollständigen Säkularisierung des Bewusstseins". In Gärtner, C., Pollack, D., \& Wohlrab-Sahr, M. (Hrsg.), Atheismus und religiöse Indifferenz. Opladen: Veröffentlichung der Sektion Religionssoziologie der Deutschen Gesellschaft für Soziologie: 339388.

———. 2004. "Sozialisation als Prozess der Krisenbewältigung". In Geulen, D. \& Veith, H. (Hrsg.), Sozialisationstheorie interdisziplinär. Aktuelle Perspektiven. Stuttgart: Lucius und Lucius: 155-183.

Plessner, H. 2004. "Der Mensch als Lebewesen". In Plessner, H. (Hrsg.), Mit anderen Augen. Aspekte einer philosophischen Anthropologie. Stuttgart: Reclam.

Tomasello, M. 2010. Warum wir kooperieren. Frankfurt am Main: Suhrkamp.

Weber, M. 1991. Die protestantische Ethik. Eine Aufsatzsammlung. Gütersloh: Siebenstern.

Zizek, B. 2012. Probleme und Formationen des modernen Subjekts. Zu einer Theorie universaler Bezogenheiten. Wiesbaden: VS-Verlag.

. 2013. "Handling Probation-Seekers - With a New Image of Humanity Towards a Positive Education". In Nowak, E., Schrader, D., \& Zizek, B. (Eds.), Educating Competencies for Democracy. Bern - Frankfurt am Main - New York: Peter Lang.

———. 2015a. "Der Mensch als Bewährungssucher - Versuch einer systematischen Einführung des Begriffs der Bewährung in die Sozialwissenschaft". In Garz, D. \& Zizek, B. (Hrsg.), Wie wir zu dem werden, was wir sind: Sozialisations-, biographie- und bildungstheoretische Aspekte. Wiesbaden: VS-Verlag.

- - . 2015b. "Exemplarische Rekonstruktion der Eröffnungsphase von Unterricht. Sozialisations-, bewährungs- und professionalisierungstheoretische Perspektiven auf Schule". Zeitschrift für Soziologie der Erziehung und Sozialisation ZSE 3 (2015): 321-340. 
Rekonstruktion der biographischen Genese einer Bewährungsfigur - Ein Beitrag zu einem sozialisationstheoretischen Begriff der Bewährung 
Boris Zizek (Mainz)

Rekonstruktion der biographischen Genese einer Bewährungsfigur - Ein Beitrag zu einem sozialisationstheoretischen Begriff der Bewährung

\begin{abstract}
Ziel des vorliegenden Beitrags ist es, exemplarisch die biographische Genese einer Bewährungsfigur zu rekonstruieren und auf diesem Wege zur weiteren Differenzierung dieses sozialisationstheoretischen Begriffs beizutragen. Bewährungsfiguren erweisen sich in der exemplarischen Interviewanalyse als lebensgeschichtlich erste Selbstkonturierungen, die im Verlauf des Lebens eine sukzessive Sättigung, modifizierende Ausgestaltung und eine mehr oder weniger gelungene, komplexitätserhöhende Transferierung auf neue Felder der Bewährung erfahren. Neben der Selbstvergewisserung, ist die zweite wesentliche Funktion von Bewährungsfiguren die Grundlegung der Selbstcharismatisierung, des Vertrauens in die eigenen Fähigkeiten und Problemlösungsvorschläge (Ja, das kann ich, das bin ich).
\end{abstract}

Keywords: Bewährung, Identität, Motivation

Doi: $10.14746 /$ eip.2014.2.5 